\title{
EFEITO DO PH NA ADSORÇÃO DO CORANTE AMARELO TARTRAZINA POR QUITOSANA E QUITOSANA MODIFICADA COM CIANOGUANIDINA
}

\author{
J. O. GONÇALVES ${ }^{1}$, C. F. ALVES ${ }^{1}$, T. GIORDANI ${ }^{1}$, C. N. GROTH ${ }^{1}$, M. T. SCALCO ${ }^{1}$, G. L. \\ DOTTO $^{2}$ e L. A. A. PINTO ${ }^{1}$ \\ ${ }^{1}$ Universidade Federal do Rio Grande, Escola de Química e Alimentos \\ ${ }^{2}$ Universidade Federal de Santa Maria, Departamento de Engenharia Química \\ E-mail: janaina_sde@hotmail.com
}

\begin{abstract}
RESUMO - Neste trabalho, quitosana com grau de desacetilação de $85 \%$ foi modificada com cianoguanidina, a fim de melhorar seu potencial adsorvente. Foram utilizadas técnicas de microscopia eletrônica de varredura e análise de infravermelho para comprovar a modificação. O efeito do $\mathrm{pH}(2-8)$ foi avaliado na adsorção do corante amarelo tartrazina pela quitosana pura e pela quitosana modificada. Foi observada uma banda do grupo $\mathrm{C} \equiv \mathrm{N}$ da cianoguanidina, comprovando que a mesma foi introduzida com sucesso nas cadeias poliméricas de quitosana. Verificou-se que a quitosana modificada foi o melhor adsorvente, apresentando um percentual de remoção de $70 \%$ do corante amarelo tartrazina. As máximas capacidades de adsorção foram de $430 \mathrm{mg} / \mathrm{g}$ e $255 \mathrm{mg} / \mathrm{g}$, em pH 2, para a quitosana modificada e a quitosana pura, respectivamente, mostrando que a modificação com cianoguanidina melhorou o potencial de adsorção da quitosana.
\end{abstract}

\section{INTRODUÇÃO}

Muitas indústrias utilizam corantes e pigmentos para colorir seus produtos, tendo como principal justificativa, a melhor aparência do produto (Prado e Godoy, 2003). No entanto, quando descartados de forma incorreta no ambiente, os corantes podem causar graves impactos, afetando processos simbióticos, reduzindo a capacidade de reoxigenação da água, dificultando a passagem de luz solar e, consequentemente, reduzindo a atividade fotossintética. Por isso, a remoção destes corantes antes do descarte do efluente industrial tem grande relevância do ponto de vista ambiental (Piccin et al., 2009).

A fim de encontrar técnicas alternativas para a remoção dos corantes de efluentes industriais, a adsorção recebe atenção considerável, pela vasta gama de materiais adsorventes o que têm sido desenvolvidos para esta finalidade (Ramachandra et al., 2007). A adsorção constitui um dos métodos mais simples e eficiente pelo fato de ser bastante eficaz na remoção de espécies em soluções líquidas 
em baixas concentrações, e, dependendo do material adsorvente que é utilizado, pode se tornar um método com maior custo benefício para o tratamento de efluentes (Kunz et al., 2002).

A utilização da quitosana em operações de adsorção para a remoção de corantes de soluções aquosas vem ganhando destaque na literatura, devido à presença de grupos amino e hidroxila, os quais servem como sítios ativos, fornecendo elevadas taxas de remoção (Wan Ngah et al., 2011). Além disso, podem ser realizadas modificações químicas na estrutura deste biopolímero com substâncias que possam incrementar no desempenho da quitosana como material adsorvente (Fu et al., 2004; Rinaudo, 2006).

Assim, o objetivo deste trabalho foi verificar o efeito do $\mathrm{pH}$ (2-8) na adsorção do corante amarelo tartrazina pela quitosana sem modificações e modificada com cianoguanidina.

\section{MATERIAIS E MÉTODOS}

\subsection{Adsorbato}

Neste estudo, foi utilizado o corante amarelo tartrazina que foi fornecido pela indústria Duas Rodas Ind. (Brasil) com grau de pureza de 85\%. Também é conhecido como FD\&C Yellow 5 (C.I. 19140), sendo um corante sintético pertencente ao grupo funcional dos azo-compostos. Apresenta uma massa molar 534,4 g/mol. A Figura 1 apresenta a estrutura química do corante amarelo tartrazina.<smiles>[NH3+]OC(=O)c1nn(-c2ccc(S(=O)(=O)[O-])cc2)c(O)c1N=Nc1ccc(S(=O)(=O)O[Na])cc1</smiles>

Figura 1 - Estrutura química do corante amarelo tartrazina.

Fonte: Dotto et al. (2011).

\subsection{Obtenção, caracterização e modificação da quitosana}

Os adsorventes utilizados neste trabalho foram a quitosana e a quitosana modificada. A quitosana em forma de pasta foi obtida a partir de resíduos de camarão (Penaeus brasiliensis) (Weska et al., 2007), após foi seca (Halal et al., 2010) e caracterizada de acordo com o tamanho da partícula (D), microscopia eletrônica de varredura (MEV) (Jeol, JSM-6060, Japão), massa molar (MM) (método viscosimétrico) (Zhang e Neau, 2001) e grau de desacetilação (Tolaimate et al., 2000). 
A quitosana em pó foi então modificada com cianoguanidina (Merck, 99,9\%) como se segue: $1 \mathrm{~g}$ de quitosana foi dissolvida em $100 \mathrm{~mL}$ de ácido clorídrico $1 \%$ (v/v) sob agitação. Após foram adicionados $0,53 \mathrm{~g}$ de cianoguanidina. A temperatura da reação foi aumentada para $90^{\circ} \mathrm{C}$ e a solução foi agitada durante $3 \mathrm{~h}$. A solução de quitosana modificada com cianoguanidina foi deixada em repouso até que atingisse a temperatura ambiente, após foi seca em estufa à $40^{\circ} \mathrm{C}$ por $24 \mathrm{~h}$ (Wang et al., 2013). As amostras de quitosana antes e após a modificação foram caracterizadas por microscopia eletrônica de varredura (MEV) (Jeol, JSM-6060, Japão) e análise de infravermelho (FTIR) (Prestige, 21210045, Japão), a fim de comprovar a modificação.

\subsection{Experimentos de adsorção}

As amostras de quitosana $(25 \mathrm{mg}$ ) foram adicionados a $80 \mathrm{~mL}$ de água e o $\mathrm{pH}$ foi corrigido para 2, 3, 4, 5, 6, 7 e 8 através da adição de $10 \mathrm{~mL}$ de soluções tampão fosfato dissódico/ácido cítrico 0,1 $\mathrm{mol} / \mathrm{L}$. Um volume de $10 \mathrm{~mL}$ de solução $1 \mathrm{~g} / \mathrm{L}$ de corante foram adicionados às soluções de adsorvente. Essas soluções foram colocadas em frascos de $500 \mathrm{~mL}$ e agitados a $100 \mathrm{rpm}$ utilizando um agitador termostatizado do tipo de Wagner, por 24 h à 298 K (modelo Fanem 315 SE, São Paulo , Brasil). 0 líquido foi filtrado (papel filtro Whatmann $\mathrm{n}^{\circ} 40$ ), e a quantidade de corante adsorvida foi determinada por espectrofotometria (Quimis Q108 DRM, Brasil) à $425 \mathrm{~nm}$. Os experimentos foram repetidos nas mesmas condições, utilizando a quitosana modificada com cianoguanidina. A capacidade de adsorção do corante (q) (mg/g), foi calculada de acordo com Equação 1.

$$
q=\frac{C_{0}-C_{f}}{m} V
$$

onde $C_{0}$ e $C_{f}$ as concentrações de corante inicial e final na fase líquida ( $\left.\mathrm{mg} / \mathrm{L}\right)$, respectivamente, $m$ a massa de adsorvente (g), e $V$ o volume da solução (L). O percentual de remoção $(\% R)$ foi calculado de acordo com a Equação 2.

$$
\% R=\frac{C_{0}-C_{f}}{C_{0}} \times 100
$$

\section{RESULTADOS E DISCUSSÃO}

\subsection{Caracterização da quitosana}

A Figura 2 apresenta as imagens de MEV para a quitosana sem modificação (Figura 2 (a)) e para a quitosana modificada com cianoguanidina (Figura (2b)). A partir da Figura 2, pode-se observar que a quitosana antes e depois da modificação apresentaram diferenças na sua superfície, sendo que a quitosana após a modificação apresentou uma superfície com mais concavidades e protuberâncias comparada a quitosana antes da modificação que se apresentou mais lisa. 


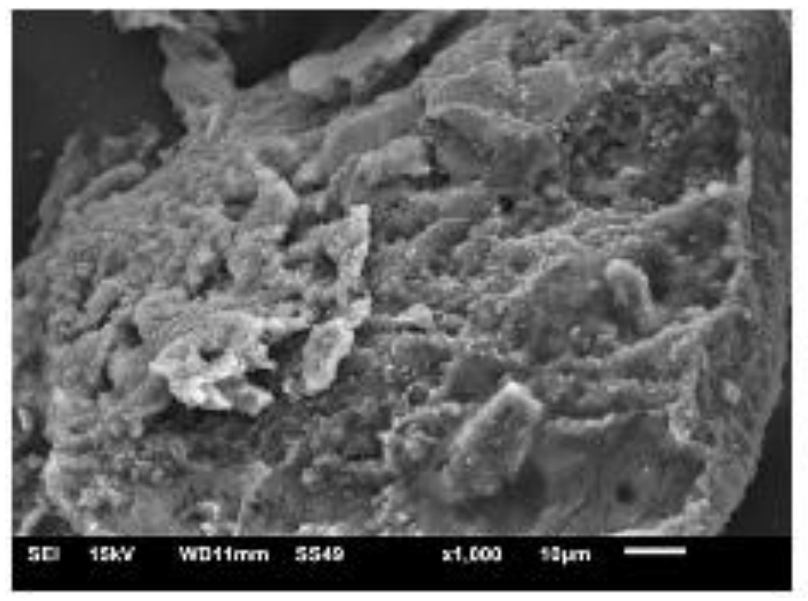

(a)

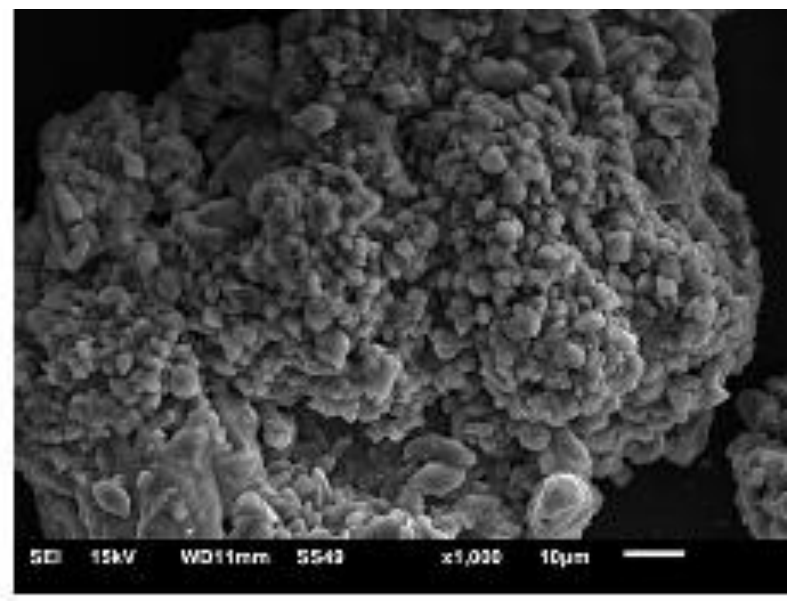

(b)

Figura 2 - Imagens de MEV para (a) quitosana sem modificação (b) quitosana modificada.

A quitosana em pó apresentou um diâmetro menor $100 \mu \mathrm{m}$, massa molar de $144 \pm 7$ kDa, e grau de desacetilação de $84,5 \pm 1 \%$. Os valores obtidos estão coerentes com o estudo recente encontrados por Dotto et al. 2013. A Figura 3 apresenta o espectro FT-IR da quitosana sem modificação e modificada.
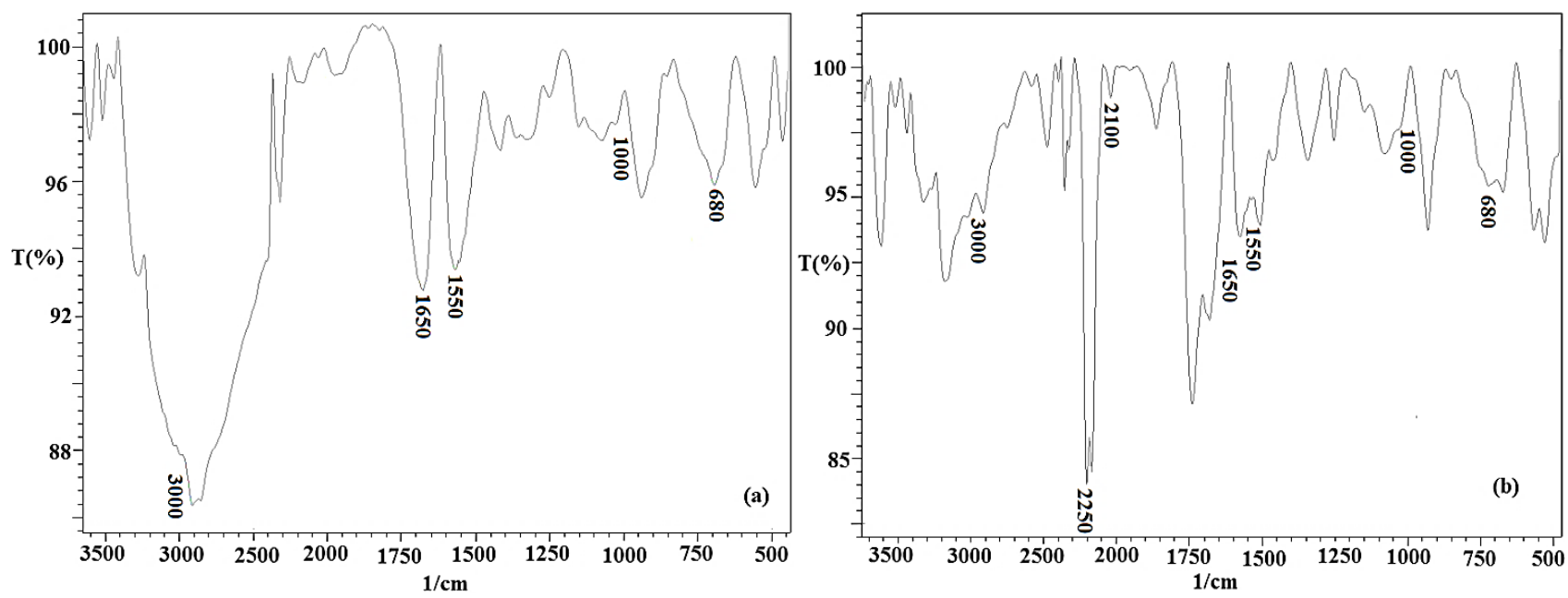

Figura 3 - Espetro infravermelho (FT-IR) (a) quitosana sem modificação (b) quitosana modificada.

Na Figura 3(a) pode se observar as bandas características da quitosana sem modificação, em $3000 \mathrm{~cm}^{-1}$, a banda larga representa os estiramentos das ligações $\mathrm{O}-\mathrm{H}$ e $\mathrm{N}-\mathrm{H}$. A vibração de $\mathrm{C}=\mathrm{O}$ (banda amida I) foi observada em $1650 \mathrm{~cm}^{-1}$. No pico $1550 \mathrm{~cm}^{-1}$, é observado o estiramento da 
ligação $\mathrm{C}-\mathrm{N}$ da amida. $\mathrm{O}$ estiramento da ligação $\mathrm{C}-\mathrm{O}$ pode ser identificado em $1000 \mathrm{~cm}^{-1}$ (Dotto et al., 2013). A deformação angular da ligação $\mathrm{N}-\mathrm{H}$ pode ser verificada em $680 \mathrm{~cm}^{-1}$ (Cadaval Jr. et al., 2013) . Após a modificação com cianoguanidina (Figura 3(b)), apareceu uma intensa banda em torno de $2250 \mathrm{~cm}^{-1}$. Este é um vínculo de sal de amônio formado (Zhao et al., 2012). Um pequeno grupo C $\equiv \mathrm{N}$ da cianoguanidina apareceu no pico $2100 \mathrm{~cm}^{-1}$. Além disso, a banda larga em torno de $3000 \mathrm{~cm}^{-1}$ diminuiu devido à inserção da cianoguanidina no grupo amino de quitosana.

A Figura 4 apresenta a influência do $\mathrm{pH}$ nas capacidades de adsorção para a quitosana sem modificação $(q)$ e modificada $\left(q_{M}\right)$.

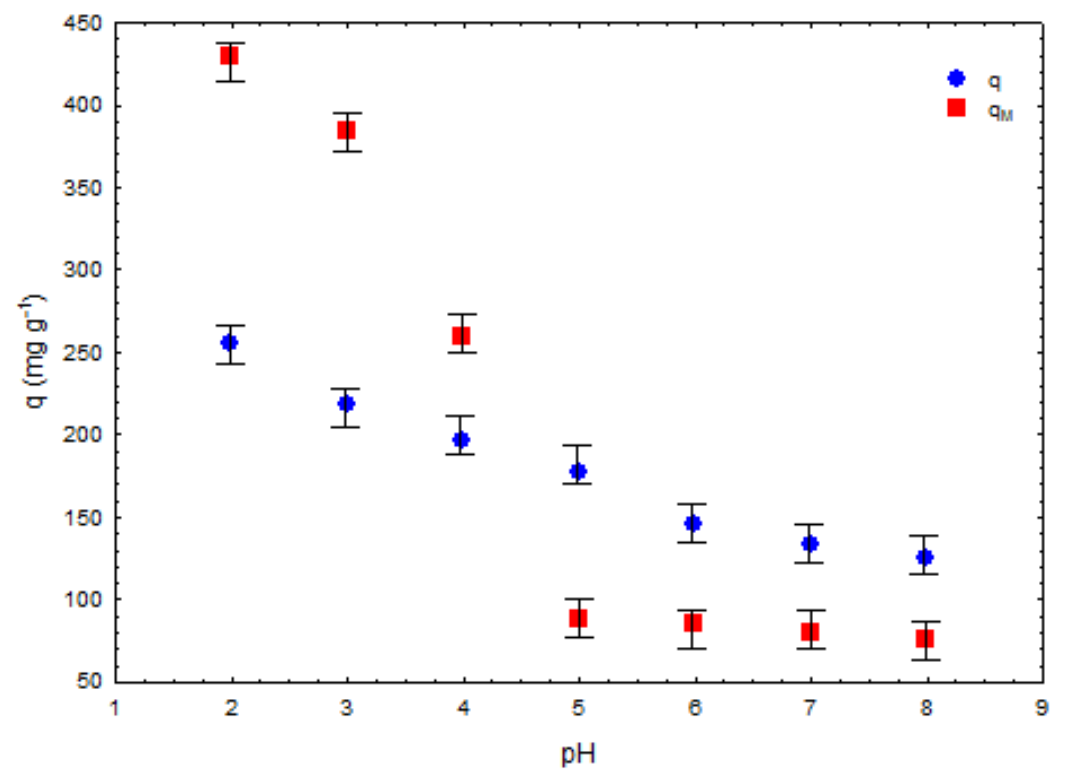

Figura 4 - Capacidades de adsorção da quitosana sem modificação $(q)$ e com modificação $\left(\mathrm{q}_{\mathrm{M}}\right)$.

Pode-se observar na Figura 4 que a quitosana e a quitosana modificada aumentaram suas capacidade de adsorção com a diminuição do $\mathrm{pH}$. Este aumento sob condições ácidas pode ter ocorrido devido ao aumento de íons $\mathrm{H}^{+}$presentes na solução, que facilitam a protonação dos grupos amino da quitosana, os quais são convertidos em $\mathrm{NH}_{3}{ }^{+}$. Estes grupamentos por sua vez promovem um aumento na interação eletrostática entre a quitosana e os grupamentos sulfonados do corante e, consequentemente, a capacidade de adsorção é aumentada (Crini e Badot, 2008; Dotto e Pinto, 2011). O percentual de remoção do corante amarelo tartrazina alcançou valores de $70 \%$.

Quanto a capacidade de adsorção da quitosana modificada com cianoguanidina, esta aumentou consideravelmente em relação à quitosana sem modificação em $\mathrm{pH}$ menores que 4,0 , com valores superiores a $260 \mathrm{mg} \mathrm{g}^{-1}$; sendo alcançado o maior valor $(430 \mathrm{mg} / \mathrm{g}) \mathrm{em} \mathrm{pH} \mathrm{2.} \mathrm{Enquanto} \mathrm{que} \mathrm{a}$ quitosana sem modificação, na mesmas condições de $\mathrm{pH}$, apresentou capacidades de adsorção na faixa de $200-255 \mathrm{mg} / \mathrm{g}$. Esse aumento deve-se a inserção da cianoguanidina nas cadeias da quitosana, o que leva a um aumento nos grupamentos carregados positivamente em meio ácido. 


\section{9 a 22 de outubro de 2014 \\ Florianópolis/SC}

\section{CONCLUSÃO}

As análises de MEV e FT-IR permitiram observar as diferenças entre a quitosana com e sem modificação, e classificar as principais vibrações características dos grupos funcionais. Foi verificado através da ligação $\mathrm{C} \equiv \mathrm{N}$ da cianoguanidina, que a quitosana foi modificada adequadamente. Mediante os ensaios de adsorção do corante amarelo tartrazina, ficou evidenciado que a modificação da quitosana com cianoguanidina favoreceu o processo de adsorção, obtendo um aumento na capacidade de adsorção de 255 para $430 \mathrm{mg} / \mathrm{g}$ no pH 2. O percentual de remoção foi de $70 \%$ do corante amarelo tartrazina. Em geral, os resultados mostraram que a modificação com cianoguanidina melhorou o potencial de adsorção da quitosana.

\section{REFERÊNCIAS BIBLIOGRÁFICAS}

CADAVAL JR., T.R.S.; CAMARA, A.S.; DOTTO, G.L.; PINTO, L.A.A. Adsorption of Cr (VI) by chitosan with different deacetylation degrees. Desalin. Water Treat., v. 51, p. 76907699, 2013.

CRINI, G.; BADOT, P. Application of chitosan, a natural aminopolysaccharide, for dye removal from aqueous solutions by adsorption processes using batch studies: A review of recent literature. Prog. Polym. Sci., v. 33, p. 399-447, 2008.

DOTTO, G.L.; MOURA, J.M.; CADAVAL Jr., T.R.S.; PINTO, L.A.A. Application of chitosan films for the removal of food dyes from aqueous solutions by adsorption. Chem. Eng. J., v. 214, p. 8-16, 2013.

DOTTO, G.L.; PINTO, L.A.A. Adsorption of food dyes acid blue 9 and food yellow 3 onto chitosan: Stirring rate effect in kinetics and mechanism. J. Hazard. Mater., v. 187, p. 164$170,2011$.

DOTTO, G.L.; VIEIRA, M.L.G.; GONÇALVES, J.O.; PINTO L.A.A. Remoção dos corantes azul brilhante, amarelo crepúsculo e amarelo tartrazina de soluções aquosas utilizando carvão ativado, terra ativada, terra diatomácea, quitina e quitosana: estudos de equilíbrio e termodinâmica, Quím. Nova, v. 34, p. 1193-1199, 2011.

FU, G.Q.; SHI, K.Y.; YUAN, Z.; NIU, W.Q.; HE, B.L.; LIU, B. A modified chitosan adsorbent for selective removal of low density lipoprotein. Chin. Chem. Let., Amsterdam, v. 15, n. 3, p. 347-349, 2004.

HALAL C.Y., MOURA J. M., PINTO L.A.A. Evaluation of molecular weight of chitosan in thin-layer and spouted bed drying, J. Food Proc., v. 34, p. 160-174, 2011.

KUNZ, A.; PERAlTA-ZAMOTRA, P.; MORAES, S.G.; DURÁN, N. Novas tendências no tratamento de efluentes têxteis. Quím. Nova, São Paulo, v. 25, n. 1, p. 78-82, 2002.

PICCIN, J.S.; VIEIRA, M.L.G.; GONÇALVES, J.; DOTTO, G.L.; PINTO, L.A.A. Adsorption of FD\&C Red No. 40 by chitosan: Isotherms analysis J. Food Eng. v. 95, p. 16-20, 2009.

RAMACHANDRA, T.V.; AHALYA, N.; KANAMADI, R.D. Biosorption: Techniques and Mechanisms. CES Technical Report, v. 110, 2007.

RINAUldO, M. Chitin and chitosan: Properties and applications. Prog. Polym. Sci.. v. 31, p. 603-632, 2006. 
TOLAIMATE, A.; DESBRIERES, J.; RHAZI, M.; ALAGUI, A.; VINCENDON, M.; VOTTERO, P. On the Influence of Deacetylation Process on the Physicochemical Characteristics of Chitosan from Squid Chitin. Polymer, v. 41, p. 256-269, 2000.

WAN NGAH, W.S.; TEONG, L.C.; HANAFIAH, M.A.K.M. Adsorption of dyes and heavy metal ions by chitosan composites: A review. Carbohydr. Polym., v. 83, p. 1446-1456, 2011.

WANG, Y.; QI, Y.; LI, Y.; WU, J.; MA, X.; YU, C.; JI L. Preparation and characterization of a novel nano-absorbent based on multi-cyanoguanidine modified magnetic chitosan and its highly effective recovery for $\mathrm{Hg}$ (II) in aqueous phase. J. Hazard. Mater., v. 260, p. 9-15, 2013.

WESKA, R.F.; MOURA, J.M.; BATISTA, L.M.; RIZZI, J.; PINTO, L.A.A. Optimization of deacetylation in the production of chitosan from shrimp wastes: use of response surface methodology, J. Food Eng., v. 80, p. 749-753, 2007.

ZHANG, H.; NEAU S.H. In vitro degradation of chitosan by a commercial enzyme preparation: effect of molecular weight and degree of deacetylation, Biomaterials, v. 22, p. 1653-1658, 2011.

ZHAO, X.; QIAO, Z.Z.; HE, J. X. Preparation of chitosan biguanidine hydrochloride and application in antimicrobial finish of wool fabric. J. Eng. Fiber. Fabr., v. 5, p. 3-20, 2012. 